\title{
Quantum automata, braid group and link polynomials
}

\author{
Silvano Garnerone
}

Dipartimento di Fisica, Politecnico di Torino, corso Duca degli Abruzzi 24, 10129 Torino (Italy)

E-mail: silvano.garnerone@polito.it

\section{Annalisa Marzuoli}

Dipartimento di Fisica Nucleare e Teorica, Università degli Studi di Pavia and Istituto Nazionale di Fisica Nucleare, Sezione di Pavia, via A. Bassi 6, 27100 Pavia (Italy)

E-mail: annalisa.marzuoli@pv.infn.it

\section{Mario Rasetti}

Dipartimento di Fisica, Politecnico di Torino, corso Duca degli Abruzzi 24, 10129 Torino (Italy)

E-mail: mario.rasetti@polito.it

\begin{abstract}
The spin-network quantum simulator model, which essentially encodes the (quantum deformed) $S U(2)$ Racah-Wigner tensor algebra, is particularly suitable to address problems arising in low dimensional topology and group theory. In this combinatorial framework we implement families of finite-states and discrete-time quantum automata capable of accepting the language generated by the braid group, and whose transition amplitudes are colored Jones polynomials. The automaton calculation of the polynomial of (the plat closure of) a link $L$ on $2 N$ strands at any fixed root of unity is shown to be bounded from above by a linear function of the number of crossings of the link, on the one hand, and polynomially bounded in terms of the braid index $2 N$, on the other. The growth rate of the time complexity function in terms of the integer $k$ appearing in the root of unity $q$ can be estimated to be (polynomially) bounded by resorting to the field theoretical background given by the Chern-Simons theory.
\end{abstract}

Key words: link invariants; braid group representations; Chern-Simons theory; quantum automata; Racah-Wigner algebra; spin-network simulator; topological quantum computation; $U_{q}(s u(2))$ representation theory. 


\section{Introduction}

The spin-network quantum simulator model [1, 2] represents a bridge between circuit schemes for standard quantum computation and approaches based on notions from Topological Quantum Field Theories (TQFT) [3, 4, 5]. The spin-network computational space, naturally modelled as a graph for any fixed number of incoming spins, supports computing processes represented by families of paths and provides, on the one hand, a consistent discretized version of the topological quantum computation approach. On the other hand, such a quantum combinatorial scheme, which essentially encodes the (quantum deformed) $S U(2)$ Racah-Wigner tensor algebra, turns out to be particularly suitable to address problems arising in (low dimensional) topology and group theory. The guiding idea of this paper is that the exponential efficiency that quantum algorithms may achieve with respect to classical ones proves to be especially relevant in problems in which the space of solutions is characterized by a structure definable in terms of the grammar and the syntax of a language, rather than algebraic or number-theoretic in nature. The spin-network setting provides a 'natural encoding' for classes of problems which basically share the combinatorial structure of the language underlying the (re)coupling theory of $S U(2)$ angular momenta [6].

On the other hand, the Jones polynomial [7] is no doubt the most famous knot invariant in topology, a knot invariant being a function on knots (or links, namely circles embedded in 3-space) which is invariant under isotopy (smooth deformations) of the knot. Among its many connections to various mathematical and physical areas (see e.g. [8] for applications in statistical mechanics), we are mainly interested here in its relations with TQFT [9]. In the seminal paper [10], Witten put link invariants in a field theoretical setting, showing that Jones polynomials arise as vacuum expectation values of Wilson loop operators in a three dimensional $S U(2)$ Chern-Simons (topological) quantum field theory where the fundamental representation of the gauge group $S U(2)$ lives on each component of the link. Such an invariant was extended to arbitrary representations living on the link components and in this paper we shall deal with such generalizations, referred to as 'extended' or 'colored' Jones polynomials [11, 12].

From the (classical) computational side, it was proved that the exact evaluation of the Jones polynomial of a link $L, V(L, \omega)$ at $\omega=$ root of unity, can be performed in polynomial time in terms of the number of crossings of the planar diagram of $L$ if $\omega$ is a 2 nd, 3rd, 4 th, 6th root of unity. Otherwise, 
the problem is $\# \mathbf{P}$-hard [13] (the computational complexity class $\# \mathbf{P}$-hard is the enumerative analog of the NP class). However, Kitaev, Larsen, Freedman and Wang [4] showed that their 'topological' quantum computation setting, relying on the same TQFT quoted before, implicitly provides an efficient quantum algorithm for the approximation of the Jones polynomial at a fifth root of unity. Unfortunately, this important algorithm was never explicitly formulated. This is particularly unfortunate since it is known that the approximation problem is $\mathbf{B Q P}$-hard, and a quantum algorithm for this problem is thus of particular importance.

Let us point out that recently Aharonov, Jones and Landau proposed an efficient quantum algorithm that approximates the problem of evaluating the Jones polynomial based, rather than on physical results from TQFT, on the path model representation of the braid group and the uniqueness of the Markov trace for the Temperley-Lieb algebra [14. The argument is that the $\# \mathbf{P}$-hardness of the problem does not rule out the possibility of good approximations, and indeed these authors provide an efficient, explicit and simple quantum algorithm to approximate the Jones polynomial at all roots of unity for both the trace and the plat closures of a braid.

Our strategy is quite different from theirs, since we shall basically provide a quantum (automaton) system whose internal evolution can be controlled in such a way that its probability amplitude gives the desired polynomial.

As mentioned, one of the features of the Jones polynomial that will be used extensively is that it can also be defined via braids (a geometric $N$ braid is a set of $N$ strands with fixed endpoints in the plane). A braid can be 'closed up' to form a link by tying its ends together. In this paper we shall be interested in one of the two ways to perform such closures, namely the plat closure of the braid, and hence consider extended Jones polynomials associated with such link diagrams, cfr. Fig. 1.

On a broader front, the study of braid groups and their applications is a field which has attracted great interest from physicists, mathematicians and computer scientists alike ( $c f r$. [15] for an updated review). Besides for its value in studying the braids in a theoretical framework, applications to knot theory have been known for years, while applicability to the field of cryptography has been realized recently [16. The analysis of algorithmic problems related to braid group has thus acquired a great practical significance, in addition to its intrinsic theoretical interest.

The approach we present here exploits a $q$-braided version of the original spin-network setting [2] to make it accept the language of the braid group 


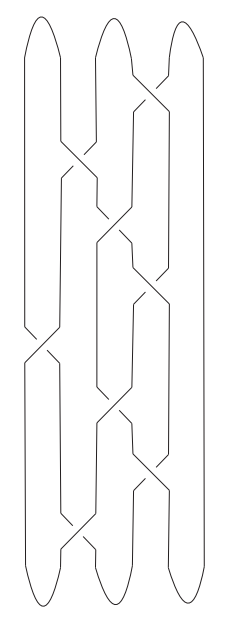

Figure 1: A plat presentation of the borromean link.

and to deal with link polynomials (see [17] for a presentation of some preliminary results). As pointed out before, the 'physical' background provided by the $3 D$ quantum $S U(2)$ Chern-Simons field theory plays a prominent role, because our computational scheme is actually designed as a discretized conterpart of the topological quantum computation setting proposed in [5]. Moreover, this framework is exactly what is needed to deal with (normalized) $S U(2)$-colored link polynomials expressed as vacuuum expectation values of composite Wilson loop operators, on the one hand, and with unitary representations of the braid group, on the other. These expectation values, in turn, will provide a bridge between the theory of formal languages and quantum computation, once more having as natural arena for discussion the $q$-braided spin-network environment. We are going to implement families of finite states (and discrete time)-quantum automata capable of accepting the language generated by the braid group, and whose transition amplitudes are colored Jones polynomials. More precisely, our results will be interpreted in terms of 'processing of words' -written in the alphabet given by the generators of the braid group- on a quantum automaton in such a way that the expectation value associated with the internal automaton 'evolution' is exactly the extended Jones polynomial. The quantum automaton in question will in turn correspond to a path in the $q$-braided spin-network computational graph. The calculation of the polynomial of (the plat closure of) a link $L$ on $2 N$ strands will be shown to be bounded from above by a linear 
function of the number of crossings of the link, on the one hand, and polynomially bounded in terms of the braid index $2 N$, on the other. Notice that the growth rate of the time complexity function in terms of the integer $k$ appearing in the root of unity $q$ can be easily estimated to be (polynomially) bounded by resorting to the TQFT background, since $k$ is nothing but the Chern-Simons coupling constant.

We shall leave as open problems the analysis of the complexity of the preparation of (initial and final) states as well as the efficient implementation of the individual automaton transition functions, which might be addressed by means of approximating (classical or quantum) algorithms.

In conclusion, we argue that our field theoretical approach could be further generalized, by suitable modifications of the braiding prescriptions in the spin-network scheme, to deal with 2 -variables link polynomials such as the HOMFLY invariant [18, related to the partition function of Potts model [8].

The content of the paper is, as far as possible, self contained. In section 2 we briefly recall the definitions of classical and quantum languages and finite states-automata. In section 3 we give a review of the spin-network computational framework modelled on the Racah-Wigner tensor algebra of $S U(2)$. In section 4 we deal with the $q$-braided version of the spin-network simulator, which relies on the tensor algebra of $U_{q}(s u(2))$ (at $q=$ root of unity). Section 5 is splitted into two parts: in 5.1 we review the 'quantum group approach' (and related $R$-matrix) to the study of (unitary) braid group representations and 'quantum' link invariants; in 5.2 we present the field-theoretical background (Chern-Simons TQFT, Wess-Zumino boundary theory, composite Wilson loop operators and their expectation values) trying to resort to geometric intuition rather than to a deep knowledge of techniques in quantum field theory. In section 6 we explain in details the automaton calculation of the extended Jones polynomial.

\section{Classical and quantum formal languages}

The theory of automata and formal languages addresses in a rigorous way the notions of computing machines and computational processes. If $A$ is an alphabet, made of letters, digits or other symbols, and $A^{*}$ denotes the set of all finite sequences of words over $A$, a language $\mathcal{L}$ over $A$ is a subset of $A^{*}$. The length of the word $w$ is denoted by $|w|$ and $w_{i}$ is its $i$ 'th symbol. The 
empty word is $\emptyset$ and the concatenation of two words $u, v$ is denoted simply by $u v$. In the sixties Noam Chomsky introduced a four level-hierarchy describing formal languages according to their structure (grammar and syntax): regular languages, context-free languages, context-sensitive languages and recursively enumerable languages. The processing of each language is inherently related to a particular computing model (see e.g. [19] for an account on formal languages). Here we are interested in finite states-automata, the machines able to accept regular languages.

A deterministic finite state automaton (DFA) consists of a finite set of states $S$, an input alphabet $A$, a transition function $F: S \times A \rightarrow S$, an initial state $s_{i n}$ and a set of accepted states $S_{a c c} \subset S$. The automaton starts in $s_{i n}$ and reads an input word $w$ from left to right. At the $i$-th step, if the automaton reads the word $w_{i}$, then it updates its state to $s^{\prime}=F\left(s, w_{i}\right)$, where $s$ is the state of the automaton reading $w_{i}$. One says that the word has been accepted if the final state reached after reading $w$ is in $S_{a c c}$.

In the case of a non-deterministic finite state automaton (NFA), the transition function is defined as a map $F: S \times A \rightarrow P(S)$, where $P(S)$ is the power set of $S$. After reading a particular symbol, the transition can lead to different states, according to some assigned probability distribution . If a NFA has $n$ states, for each symbol $a \in A$ there is an $n \times n$ transition matrix $M_{a}$ for which $\left(M_{a}\right)_{i j}=1$ if and only if the transition from the state $i$ to the state $j$ is allowed once the symbol $a$ has been read.

Generally speaking, quantum finite states-automata (QFA) are obtained from their classical probabilistic counterparts by moving from the notion of (classical) probability associated with transitions to quantum probability amplitudes. Computation takes place inside a suitable Hilbert space through unitary matrices and a number of different models have been proposed, see e.g. 20, 21], just to mention a couple of them. Following [21], the measureonce quantum automaton is a 5-tuple $M=\left(Q, \Sigma, \delta, \mathbf{q}_{0}, \mathbf{q}_{f}\right)$, where $Q$ is a finite set of states, $\Sigma$ is a finite input alphabet with an end-marker symbol $\#$ and $\delta: Q \times \Sigma \rightarrow Q$ is the transition function. Here $\delta\left(\mathbf{q}, \sigma, \mathbf{q}^{\prime}\right)$ is the probability amplitude for the transition from the state $\mathbf{q}$ to the state $\mathbf{q}^{\prime}$ upon reading the symbol $\sigma$. The state $\mathbf{q}_{0}$ is the initial configuration of the system, and $\mathbf{q}_{f}$ is an accepted final states. For all states and symbols the function $\delta$ must be unitary. The end-marker \# is the last symbol of each input and computation terminates after reading it. At the end of the computation the automaton measures its configuration: if it is an accepted state then the input is accepted, otherwise is rejected. The configuration of 
the automaton is in general a superposition of states in the Hilbert space where the automaton lives. The transition function is represented by a set of unitary matrices $U_{\sigma}(\sigma \in \Sigma)$, where $U_{\sigma}$ represents the unitary transition of the automaton reading the symbol $\sigma$. The probability amplitude for the automaton of accepting the string $w$ is given by

$$
f_{M}(w)=\left\langle\mathbf{q}_{f}\left|U_{w}\right| \mathbf{q}_{0}\right\rangle,
$$

and the explicit form of $f_{M}(w)$ defines the language $\mathcal{L}$ accepted by that particular automaton. If $\hat{P}$ denotes the projector over the accepted states, the probability for the automaton of accepting the string $w$ is given by

$$
p_{M}(w)=\| \hat{P}\left|\psi_{w}\right\rangle \|^{2}
$$

where $\left|\psi_{w}\right\rangle \doteq U_{w}\left|\mathbf{q}_{0}\right\rangle$.

\section{The quantum spin-network simulator}

The spin network model of computation was introduced in [1] and worked out in [2] as a general framework for processing information in the quantum context and is essentially modelled on the combinatorics of the RacahWigner algebra of $S U(2)$. The spin-network can be seen as a collection of graphs $\mathfrak{G}_{n}(V, E)$ parametrized by an integer $n(n \geq 2)$, where $n+1$ is the number of incoming angular momentum variables, each associated with an irreducible representation (irrep) of $S U(2),\left\{j_{i}\right\} \in\{0,1 / 2,1,3 / 2 \ldots\}$ in $\hbar$ units (we choose units in which $\hbar=1$ ). On the physical side, these $n+1$ basic variables enter in the construction of different sets of (pure angular momenta) eigenspaces selected according to the different types of quantum interactions we whish to simulate. The fact that physical interactions in many (conservative) quantum systems can be well modelled on (combinations of) two-body interactions 22 opens the possibility of calling into play the powerful algebraic-combinatorial setting underlying $S U(2)$ binary coupling and recoupling theory ( $c f r$. [6] and the original references therein).

Before going into some more details on this realization of the spin-network graphs, let us point out that the combinatorial structure encoded into the Racah-Wigner algebra is actually shared by other discrete structures.

A first type of realization is purely graph-theoretical. The vertex set $V$ of the graph $\mathfrak{G}_{n}(V, E)$ can be identified with the set of (rooted) binary trees 
with $n+1$ labelled leaves where the leaves (terminal nodes) and the internal nodes of the trees are labelled by integers and half-integers $\in \frac{1}{2} \mathbb{N}$, cfr. Fig. 2. Undirected edges between vertices are drawn whenever a pair of vertices

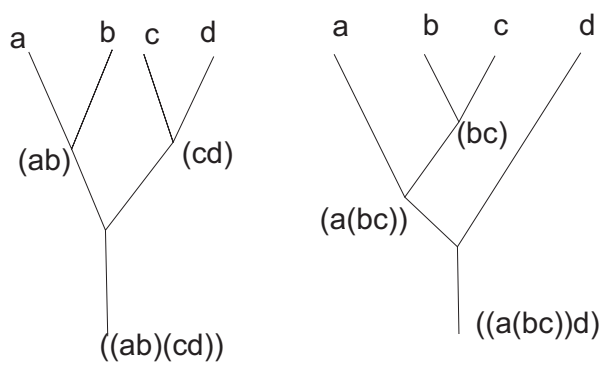

Figure 2: Two labelled binary trees on $(n+1)=4$ leaves. Such trees are in one-to-one correspondence with the vertex set $V$ of the graph $\mathfrak{G}_{3}(V, E)$

(labelled trees) are connected by two kinds of topological elementary moves, namely twist and rotation, illustrated in Fig. [3.

The resulting graph, known as Twist-Rotation graph, is depicted for $n+1=4$ in Fig. 4 and its combinatorial properties are analyzed in [23] and in Appendix A of [2].

Another realization of the spin-network is in terms of words endowed with pairs of parentheses representing a non-commutative and non-associative binary operation. In this case the vertices of the graph $\mathfrak{G}_{n}(V, E)$ are associated with words $w$ made of letters from the alphabet $\left\{\frac{1}{2} \mathbb{N} \cup\right.$ pairs of labelled parentheses $\left.(\cdot \cdot)_{a}\right\}$, e.g.

$$
w=\left(\left(\left(j_{1}, j_{2}\right)_{k_{1}}, j_{3}\right)_{k_{2}}, \ldots\right)_{J} ; j_{i}, k_{l} \in \frac{1}{2} \mathbb{N} \text { with } j_{1}+j_{2}+\ldots j_{n+1}=J,
$$

where $J$ is the label assigned to the root. Two vertices are connected by an edge if it is possible to switch from one to the other either by swapping the elements inside a parenthesis, $(a, b)_{c} \leftrightarrow(b, a)_{c}$, or by changing the parenthesization structure $\left((\cdot, \cdot)_{k_{1}}, \cdot\right)_{k_{2}} \leftrightarrow\left(\cdot,(\cdot, \cdot)_{h_{1}}\right)_{h_{2}}$.

Coming back to the Racah-Wigner setting, the interpretation of the spinnetwork graph goes on as follows. There exists a one-to-one correspondence $\{v(\mathfrak{b})\} \longleftrightarrow\left\{\mathcal{H}_{n}^{J}(\mathfrak{b})\right\}$ between the vertices of $\mathfrak{G}_{n}(V, E)$ and the computational Hilbert spaces of the simulator. The label $\mathfrak{b}$ has the following meaning: for any given pair $(n, J)$, all binary coupling schemes of the $n+1$ angular momenta $\left\{\mathbf{J}_{\ell}\right\}$, identified by the quantum numbers $j_{1}, \ldots, j_{n+1}$ (summing 


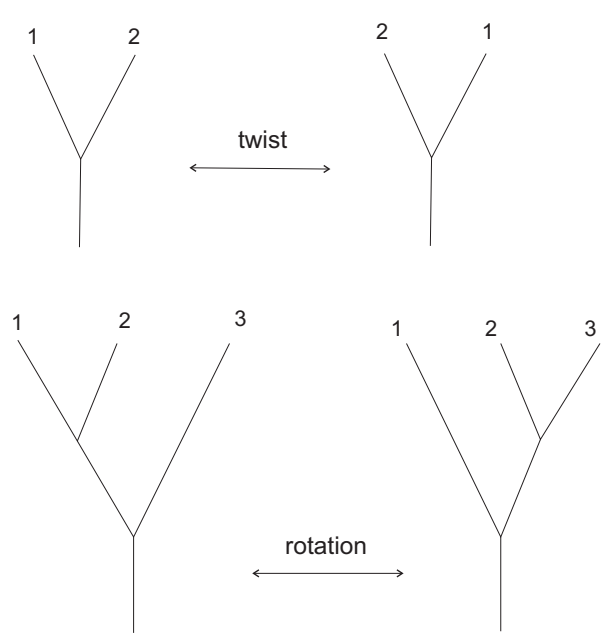

Figure 3: A twist corresponds to the interchange of either two leaves or two subtrees (top). A rotation consists in a change of the coupling scheme of either three leaves or subtrees (bottom).

up to a total $J$ ) plus $k_{1}, \ldots, k_{n-1}$ (corresponding to the $n-1$ intermediate angular momenta $\left\{\mathbf{K}_{i}\right\}$ ) and by the brackets defining the binary couplings, provide the 'alphabet' in which quantum information is encoded (the rules and constraints of bracketing are instead part of the 'syntax' of the resulting coding language). The Hilbert spaces $\mathcal{H}_{n}^{J}(\mathfrak{b})$ thus generated are spanned by complete orthonormal sets of states with suitable quantum number label set such as, e.g. for $n=3,\left\{\left(\left(j_{1}\left(j_{2} j_{3}\right)_{k_{1}}\right)_{k_{2}} j_{4}\right)_{J},\left(\left(j_{1} j_{2}\right)_{k_{1}^{\prime}}\left(j_{3} j_{4}\right)_{k_{2}^{\prime}}\right)_{J}\right\}$.

More precisely, for a given value of $n, \mathcal{H}_{n}^{J}(\mathfrak{b})$ is the simultaneous eigenspace of the squares of $2(n+1)$ Hermitean, mutually commuting angular momentum operators $\mathbf{J}_{1}, \mathbf{J}_{2}, \mathbf{J}_{3}, \ldots, \mathbf{J}_{n+1}$ with fixed sum $\mathbf{J}_{1}+\mathbf{J}_{2}+\mathbf{J}_{3}+\ldots+\mathbf{J}_{n+1}=\mathbf{J}$, of the intermediate angular momentum operators $\mathbf{K}_{1}, \mathbf{K}_{2}, \mathbf{K}_{3}, \ldots, \mathbf{K}_{n-1}$ and of the operator $J_{z}$ (the projection of the total angular momentum $\mathbf{J}$ along the quantization axis). The associated quantum numbers are $j_{1}, j_{2}, \ldots, j_{n+1}$; $J ; k_{1}, k_{2}, \ldots, k_{n-1}$ and $M$, where $-J \leq M \leq+J$ in integer steps.

If $\mathcal{H}^{j_{1}} \otimes \mathcal{H}^{j_{2}} \otimes \cdots \otimes \mathcal{H}^{j_{n}} \otimes \mathcal{H}^{j_{n+1}}$ denotes the factorized Hilbert space, namely the $(n+1)$-fold tensor product of the individual eigenspaces of the $\left(\mathbf{J}_{\ell}\right)^{2}$ 's, the operators $\mathbf{K}_{i}$ 's represent intermediate angular momenta generated, through Clebsch-Gordan series, whenever a pair of $\mathbf{J}_{\ell}$ 's are coupled. As an example, by coupling sequentially the $\mathbf{J}_{\ell}$ 's according to the scheme $\left(\cdots\left(\left(\mathbf{J}_{1}+\right.\right.\right.$ 


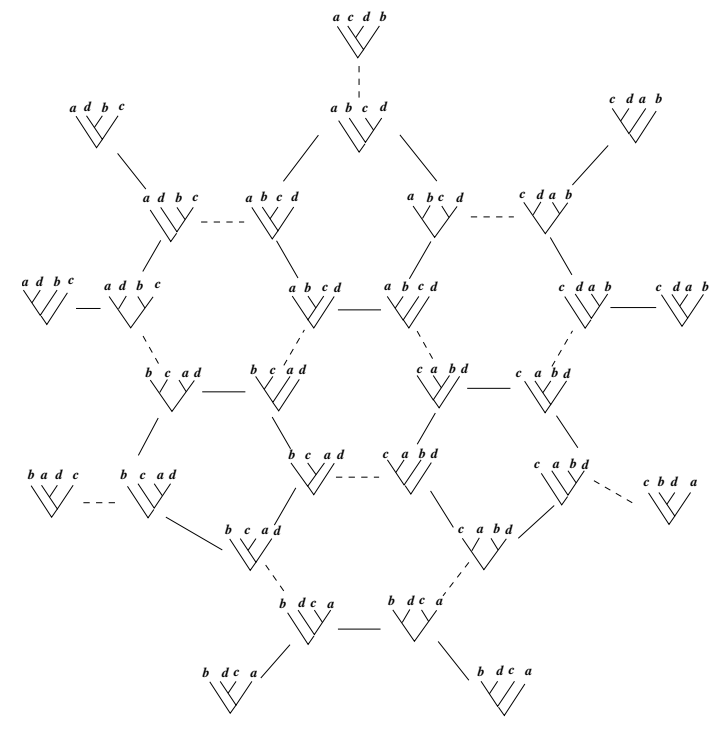

Figure 4: A portion of the Twist-Rotation graph $\mathfrak{G}_{3}(V, E)$ where only 30 out of 60 vertices are shown (the picture can be completed by taking the mirror image of each tree at the antipodal vertex). The remaining 60 vertices are arranged into an isomorphic graph obtained by swapping one pair of labels, e.g. $(a, b) \rightarrow(b, a)$. Solid edges represent rotations and dashed edges represent twists.

$\left.\left.\left.\mathbf{J}_{2}\right)+\mathbf{J}_{3}\right)+\cdots+\mathbf{J}_{n+1}\right)=\mathbf{J}-$ which generates $\left(\mathbf{J}_{1}+\mathbf{J}_{2}\right)=\mathbf{K}_{1},\left(\mathbf{K}_{1}+\mathbf{J}_{3}\right)=$ $\mathbf{K}_{2}$, and so on - we should get a binary bracketing structure of the type $\left(\cdots\left(\left(\left(\mathcal{H}^{j_{1}} \otimes \mathcal{H}^{j_{2}}\right)_{k_{1}} \otimes \mathcal{H}^{j_{3}}\right)_{k_{2}} \otimes \cdots \otimes \mathcal{H}^{j_{n+1}}\right)_{k_{n-1}}\right)_{J}$, where for completeness we add an overall bracket labelled by the quantum number of the total angular momentum $J$. Note that, as far as $j_{\ell}$ 's quantum numbers are involved, any value belonging to $\{0,1 / 2,1,3 / 2, \ldots\}$ is allowed, while the ranges of the $k_{i}$ 's are suitably constrained by Clebsch-Gordan decompositions (e.g. if $\left.\left(\mathbf{J}_{1}+\mathbf{J}_{2}\right)=\mathbf{K}_{1} \Rightarrow\left|j_{1}-j_{2}\right| \leq k_{1} \leq j_{1}+j_{2}\right)$.

We denote a binary coupled basis of $(n+1)$ angular momenta in the $J M-$ representation and the corresponding Hilbert space as

$$
\begin{gathered}
\left\{\left|\left[j_{1}, j_{2}, j_{3}, \ldots, j_{n+1}\right]^{\mathfrak{b}} ; k_{1}^{\mathfrak{b}}, k_{2}^{\mathfrak{b}}, \ldots, k_{n-1}^{\mathfrak{b}} ; J M\right\rangle,-J \leq M \leq J\right\} \\
=\mathcal{H}_{n}^{J}(\mathfrak{b}) \doteq \operatorname{span}\left\{|\mathfrak{b} ; J M\rangle_{n}\right\}
\end{gathered}
$$


where the string inside $\left[j_{1}, j_{2}, j_{3}, \ldots, j_{n+1}\right]^{\mathfrak{b}}$ is not necessarily an ordered one, $\mathfrak{b}$ indicates the current binary bracketing structure and the $k_{i}$ 's are uniquely associated with the chain of pairwise couplings selected by $\mathfrak{b}$.

For a given value of $J$ each $\mathcal{H}_{n}^{J}(\mathfrak{b})$ has dimension $(2 J+1)$ over $\mathbb{C}$, but Hilbert spaces corresponding to different bracketing schemes, although isomorphic, are not identical. They actually correspond to (partially) different complete sets of physical observables, namely for instance $\left\{\mathbf{J}_{1}^{2}, \mathbf{J}_{2}^{2}, \mathbf{J}_{12}^{2}, \mathbf{J}_{3}^{2}, \mathbf{J}^{2}, J_{z}\right\}$ and $\left\{\mathbf{J}_{1}^{2}, \mathbf{J}_{2}^{2}, \mathbf{J}_{3}^{2}, \mathbf{J}_{23}^{2}, \mathbf{J}^{2}, J_{z}\right\}$ respectively (in particular, $\mathbf{J}_{12}^{2}$ and $\mathbf{J}_{23}^{2}$ cannot be measured simultaneously). On the mathematical side this remark reflects the fact that the tensor product $\otimes$ is an associative operation only up to isomorphisms.

For what concerns unitary operations acting on the computational Hilbert spaces (4), we shall consider here unitary transformations associated with recoupling coefficients ( $3 n j$ symbols) of $S U(2)$, thought of as $j$-gates in the present quantum computing context. As shown in [6], any such coefficient can be splitted into 'elementary' $j$-gates, namely Racah and phase transforms. A Racah transform applied to a basis vector is defined formally as

$$
\mathcal{R}:\left|\ldots\left((a b)_{d} c\right)_{f} \ldots ; J M\right\rangle \mapsto\left|\ldots\left(a(b c)_{e}\right)_{f} \ldots ; J M\right\rangle
$$

where Latin letters $a, b, c, \ldots$ are used here to denote generic, both incoming ( $j_{\ell}$ 's in the previous notation) and intermediate $\left(k_{i}\right.$ 's) spin quantum numbers (this operation corresponds to a rotation in the Twist-Rotation graph, crf. Fig. 3, bottom and Fig. 4). Its explicit expression reads

$$
\begin{gathered}
\left|\left(a(b c)_{e}\right)_{f} ; M\right\rangle \\
=\sum_{d}(-1)^{a+b+c+f}[(2 d+1)(2 e+1)]^{1 / 2}\left\{\begin{array}{ccc}
a & b & d \\
c & f & e
\end{array}\right\}\left|\left((a b)_{d} c\right)_{f} ; M\right\rangle,
\end{gathered}
$$

where there appears the $6 j$ symbol of $S U(2)$ and $f$ plays the role of the total angular momentum quantum number. Note that, according to the WignerEckart theorem, the quantum number $M$ (as well as the angular part of wave functions) is not altered by such transformations, and that the same happens with any $3 n j$ symbol. On the other hand, the effect of a phase transform $\Phi$ (a twist operation on the Twist-Rotation graph, see Fig. 3, top and Fig. 
4) amounts to introducing a suitable phase whenever two spin labels are swapped

$$
\left|\ldots(a b)_{c} \ldots ; J M\right\rangle=(-1)^{a+b-c}\left|\ldots(b a)_{c} \ldots ; J M\right\rangle .
$$

These unitary operations are combinatorially encoded into the edge set $E=$ $\{e\}$ of the graph $\mathfrak{G}_{n}(V, E): E$ is just the subset of the Cartesian product $(V \times V)$ selected by the action of these unitary $j$-gates.

In the framework described above, a computation is represented in a natural way by a collection of step-by-step transition rules (gates), namely a family of 'elementary unitary operations' and we assume that it takes one unit of the intrinsic discrete time variable to perform anyone of them. Such prescriptions amount to select (families of) 'directed paths' in the spin-network computational space $\mathfrak{G}_{n}(V, E) \times \mathbb{C}^{2 J+1}$, all starting from the same input state and ending in an admissible output state. A single path in the given family can be interpreted as a (finite-states) quantum automaton calculation, once we select a particular encoding scheme for the problem we wish to address.

By a directed path $\mathcal{P}$ with fixed endpoints we mean a (time) ordered sequence

$$
\left|\mathbf{v}_{\text {in }}\right\rangle_{n} \equiv\left|\mathbf{v}_{0}\right\rangle_{n} \rightarrow\left|\mathbf{v}_{1}\right\rangle_{n} \rightarrow \cdots \rightarrow\left|\mathbf{v}_{s}\right\rangle_{n} \rightarrow \cdots \rightarrow\left|\mathbf{v}_{\mathbb{L}}\right\rangle_{n} \equiv\left|\mathbf{v}_{\text {out }}\right\rangle_{n}
$$

where we use the shorthand notation $\left|\mathbf{v}_{s}\right\rangle_{n}$ for computational states (which are vectors expressed in the bases (44) ) and $s=0,1,2, \ldots, \mathbb{L}(\mathcal{P})$ is the lexicographical labelling of the states along the path. Finally, $\mathbb{L}(\mathcal{P})$ is the length of the path $\mathcal{P}$ and $\mathbb{L}(\mathcal{P}) \cdot \tau \doteq T$ is the time required to perform the process in terms of the discrete time unit $\tau$.

A computation consists in evaluating the expectation value of the unitary operator $\mathbf{U}_{\mathcal{P}}$ associated with the path $\mathcal{P}$, namely

$$
\left\langle\mathbf{v}_{\text {out }}\left|\mathbf{U}_{\mathcal{P}}\right| \mathbf{v}_{\text {in }}\right\rangle_{n}
$$

By taking advantage of the possibility of decomposing $\mathbf{U}_{\mathcal{P}}$ uniquely into an ordered sequence of elementary gates, (9) becomes

$$
\left\langle\mathbf{v}_{\text {out }}\left|\mathbf{U}_{\mathcal{P}}\right| \mathbf{v}_{\text {in }}\right\rangle_{n}=\left\lfloor\prod_{s=0}^{\mathbb{L}-1}\left\langle\mathbf{v}_{s+1}\left|\mathcal{U}_{s, s+1}\right| \mathbf{v}_{s}\right\rangle_{n}\right\rfloor_{\mathcal{P}}
$$


with $\mathbb{L} \equiv \mathbb{L}(\mathcal{P})$ for short. The symbol $\rfloor_{\mathcal{P}}$ denotes the ordered product along the path $\mathcal{P}$ and each elementary operation is rewritten as $\mathcal{U}_{s, s+1}(s=$ $0,1,2, \ldots \mathbb{L}(\mathcal{P}))$ to stress its 'one-step' character. Such expectation values are particular instances of the general expression (11) for the quantum amplitude of a finite-states automaton, once a suitable language has been encoded into the computational space of the spin-network simulator.

\section{$4 q$-braided computational space}

As we shall see in the following section, the basic ingredient for addressing link invariants arising in the context of Chern-Simons field theory is the 'tensor structure' naturally associated with the representation ring of the Lie algebra of a simple compact group which plays the role of the gauge group of the theory. In the case of $S U(2)$ this structure is provided by (tensor products of) Hilbert spaces supporting irreducible representations together with unitary morphisms between them: these are exactly the objects collected into the Racah-Wigner algebra discussed in section 3. However, when dealing with (planar diagrams of) links we shall also have to specify the eigenvalues of the braiding matrix to be associated with the crossings of the links and this extension can be achieved by 'braiding' the Racah-Wigner tensor category. In the present context, it is natural to take advantage of quantum group techniques in order to 'split' any phase transform (77) by assigning different weights -depending on a deformation parameter $q$ to be defined below - to right and left handed twists. From the combinatorial viewpoint, this generalization corresponds to replace the spin network computational space $\mathfrak{G}_{n}(V, E) \times \mathbb{C}^{2 J+1}$ with its $q$-braided counterpart (see Fig. [5])

$$
\left(\left(\mathfrak{G}_{n}(V, E) \times \mathbb{C}^{2 J+1}\right) \times \mathbb{Z}_{2}\right)_{q}
$$

where the (classical) $6 j$ symbol in any Racah trasform (6) will become $q$ deformed.

The tensor category we are going to introduce is associated with the quantum group $U_{q}(s u(2))$ ( $q=$ root of unity), namely the universal enveloping algebra of $S U(2)$ endowed with additional structures which make it a quasitriangular quasi-Hopf-*algebra (see e.g. 24] and other references therein). $U_{q}(s u(2))$ is an associative algebra generated by elements $J_{+}, J_{-}$and $J_{z}$ which satisfy the commutation relations

$$
\left[J_{z}, J_{ \pm}\right]= \pm J_{ \pm} ;\left[J_{+}, J_{-}\right]=\left[2 J_{z}\right]_{q}
$$




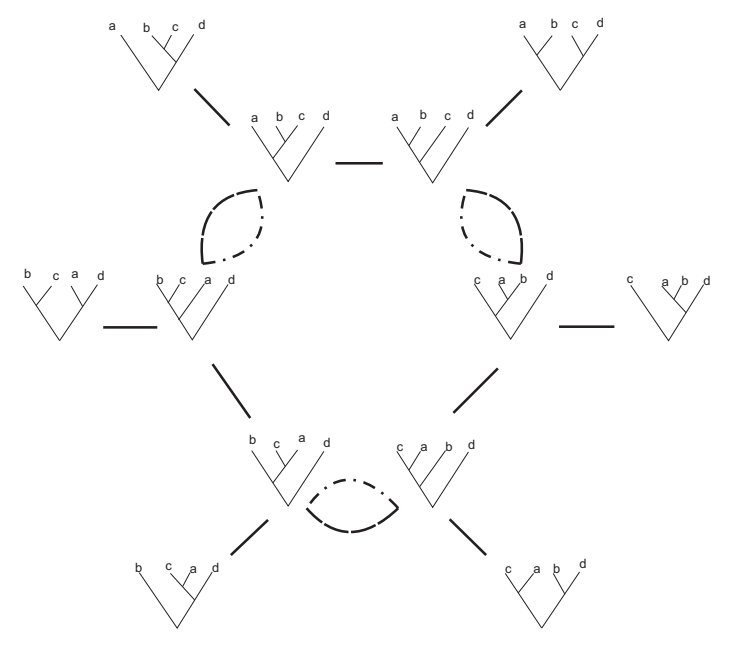

Figure 5: A portion of the $q$-braided Twist Rotation graph $\left(\mathfrak{G}_{3}(V, E) \times \mathbb{Z}_{2}\right)_{q}$ : with respect to the unbraided situation, each twist has been splitted.

where the $q$-integer $[\mathbf{n}]_{q}$ is defined as $[\mathbf{n}]_{q} \equiv\left(q^{\mathbf{n} / 2}-q^{-\mathbf{n} / 2}\right) /\left(q^{1 / 2}-q^{-1 / 2}\right)$. $U_{q}(s u(2))$ is a deformation of the universal enveloping algebra of the Lie algebra $s u(2)$ since in the limit $q \rightarrow 1$ the above relations reduce to the commutation relations for the $s u(2)$ generators.

A Hopf algebra-structure can be introduced by defining the coproduct homomorphism

$$
\Delta: U_{q}(s u(2)) \rightarrow U_{q}(s u(2)) \otimes U_{q}(s u(2)),
$$

acting on $J_{+}, J_{-}$and $J_{z}$ according to

$$
\begin{gathered}
\Delta\left(J_{ \pm}\right)=J_{ \pm} \otimes q^{\frac{J}{2}}+q^{-\frac{J}{2}} \otimes J_{ \pm} \\
\Delta\left(J_{z}\right)=J_{z} \otimes 1+1 \otimes J_{z} .
\end{gathered}
$$

The tensor algebra associated with $U_{q}(s u(2))$ can be worked out in practice as in the case of $s u(2)$, so that we have Hilbert spaces supporting irreducible representations, $q$-Clebsch-Gordan coefficients, $q$-Racah coefficients and so on. The crucial difference consists in the fact that the irreps label set acquires a cut-off, namely each label must be chosen in the set $\{0,1 / 2,1,3 / 2, \ldots$, $k-2\}$, where the integer $k$ is related to the deformation parameter $q$ by $q$ $=\exp (-2 i \pi / k)$. Denoting by $\mathcal{H}_{q}^{j_{1}}$ and $\mathcal{H}_{q}^{j_{2}}$ the Hilbert spaces supporting two 
irreps $j_{1}, j_{2}$, their (truncated) tensor product can be decomposed according to the Clebsch-Gordan series

$$
\mathcal{H}_{q}^{j_{1}} \otimes \mathcal{H}_{q}^{j_{2}}=\bigoplus_{j=\left|j_{1}-j_{2}\right|}^{\min \left\{j_{1}+j_{2}, k-j_{1}-j_{2}\right\}} \mathcal{H}_{q}^{j}
$$

As happens in the classical case, the two bases associated with the eigenspaces involved in the tensor product (13) can be connected by means of ClebschGordan coefficients according to

$$
|j m\rangle_{q}=\sum_{m_{1}, m_{2}}\left(j_{1} j_{2} m_{1} m_{2} \mid j m\right)_{q}\left|j_{1} m_{1}\right\rangle_{q}\left|j_{2} m_{2}\right\rangle_{q}
$$

where $-j_{1} \leq m_{1} \leq j_{1},-j_{2} \leq m_{2} \leq j_{2}, m=m_{1}+m_{2}$. Quantum CG coefficients ()$_{q}$ can be suitable normalized and satisfy orthogonality relations 25 .

The quantum Racah transformation comes out when we consider different binary couplings in the tensor product $\mathcal{H}_{q}^{j_{1}} \otimes \mathcal{H}_{q}^{j_{2}} \otimes \mathcal{H}_{q}^{j_{3}}$ of three irreducible representations, as done in the classical case ( $c f r$. (15) and (6) ). For instance

$$
\begin{gathered}
\left|\left(j_{1} j_{2}\right)_{j_{12}} j_{3} ; j m\right\rangle_{q} \\
=\sum_{j_{23}} W_{q}\left(j_{1} j_{2} j j_{3} ; j_{12} j_{23}\right)\left(\left[2 j_{12}+1\right]_{q}\left[2 j_{23}+1\right]_{q}\right)^{-1 / 2}\left|j_{1}\left(j_{2} j_{3}\right)_{j_{23}} ; j m\right\rangle_{q},
\end{gathered}
$$

where there appear the $q$-dimensions of the irreps involved. The components of $W_{q}$ are the Racah coefficients of the algebra $U_{q}\left(s u_{2}\right)$ and these symbols satisfy orthogonality relations, symmetry properties and identities which look like suitable $q$-deformations of the corresponding classical ones (and reduce to them in the limit $q \rightarrow 1$ ) 25]. The quantum Racah coefficient and the $q$-counterpart of the Wigner $6 j$ symbol differ as usual by a phase factor, namely

$$
W_{q}\left(j_{1} j_{2} j j_{3} ; j_{j_{12}} j_{j_{23}}\right) \doteq(-1)^{j_{1}+j_{2}+j_{3}+j}\left\{\begin{array}{ccc}
j_{1} & j_{2} & j_{12} \\
j_{3} & j & j_{23}
\end{array}\right\}_{q}
$$

Finally, we introduce the (differently normalized) symbol

$$
\left(\begin{array}{cc|c}
j_{1} & j_{2} & j_{12} \\
j_{3} & j & j_{23}
\end{array}\right)_{q} \doteq \frac{W_{q}\left(j_{1} j_{2} j j_{3} ; j_{j_{12}} j_{j_{23}}\right)}{\sqrt{\left[2 j_{12}+1\right]_{q}\left[2 j_{23}+1\right]_{q}}}
$$


which, on the one hand, enhances the matrix character of the quantum Racah transform (15) and, on the other, is particularly suitable to be generalized to deal with more than three incoming spin labels.

\section{Quantum invariants of links and unitary representations of the braid group}

Link invariants are functions on links (collections of knots, namely closed circles in 3-space) which depend only on the isotopy class of the link. An (ambient) isotopy can be thought of as a continuous transformation performed on the link embedded in $\mathbb{R}^{3}$ which deformes at will the shape of the link without cuttings. Let us point out preliminarly that the link invariants of polynomial-type we are going to address here are 'universal' in the sense that historically distinct approaches $(\mathrm{R}$-matrix representations obtained with the quantum group method, monodromy representations of the braid group in $2 D$ conformal field theories, the quasi tensor category approach by Drinfeld and the $3 D$ quantum Chern-Simons theory, see e.g. [26, 27] for reviews) are indeed different aspects of the same underlying algebraic structure. We shall focus in particular on the Chern-Simons setting [10] since, on the one hand, it embraces the universal structure of (unitary) braid group representations shared by all the models quoted above and, on the other, can be naturally encoded into the (braided) spin-network computational scheme. The (colored) link polynomials arising from $S U(2)$ quantum CS theory can be referred to as 'extended' Jones polynomials, since the Jones polynomial [7] is recovered by selecting the fundamental $\left(j=\frac{1}{2}\right)$ representation of $S U(2)$ on each of the link components (or on each strand of the associated braid). Moreover, the topological quantum field approach is inherently related to low dimensional geometry since, for instance, suitable combinations of these invariants can be interpreted as topological invariants of hyperbolic 3-manifolds, obtained by surgery along framed links in the 3-sphere [11, 12].

Let us point out that the definitions of link polynomials from Hecke (or Temperley-Lieb) algebra realizations of the braid group -exploited in [14] in the quantum computational context- can be derived quite easily in the

framework we are adopting here, since it can be shown that the associated invariants do satisfy the linear skein relations which characterize such realizations [26]. 
Before addressing a full fledged approach to $3 D$ Chern-Simons theory, we pause a little bit digressing on $R$-matrix representations of the braid group arising from quantum groups. The associated invariants of knots and links are commonly refereed to as 'quantum' invariants, since they are quantities depending on the deformation parameter $q$ of the 'quantum group' under consideration.

\subsection{The quantum group approach}

Let $\mathfrak{g}$ be a (semi)simple Lie algebra, $U_{q}(\mathfrak{g})$ its universal enveloping algebra and $V$ a finite dimensional (complex) vector space in the associated tensor algebra (the prototype is of course the unitary tensor algebra of $U_{q}(s u(2))$ described in details in section 4). The representation theory of any such quantum group is naturally endowed with an invertible linear operator, the so-called $R$-matrix

$$
R: V \otimes V \rightarrow V \otimes V,
$$

which satisfies the quantum Yang-Baxter equation

$$
(R \otimes I)(I \otimes R)(R \otimes I)=(I \otimes R)(R \otimes I)(I \otimes R),
$$

where both sides of the above expression are to be understood as linear transformations $V \otimes V \otimes V \rightarrow V \otimes V \otimes V$.

The general procedure for constructing quantum invariants of oriented knots (or links) presented as closure (or platting) of braids can be outlined as follows. Consider an oriented knot diagram (namely the projection of a knot with orientation onto a fixed plane) and insert an horizontal line as depicted in Fig. 6.

To each intersection point between the line and the diagram we assign either the representation space $V$ or its dual $V^{*}$, depending on whether the portion of the knot nearby the intersection is oriented upwards or downwards. The whole configuration of such points on the line turns out to be associated with the tensor product of the individual vector spaces (orderered from left to right). The connection with braid groups comes out when we consider two parallel horizontal lines intersecting the knot diagram. More precisely, the portion of the knot diagram between a pair of horizontal lines represents the geometric realization of a braid $b$, which in turn is an element of the Artin braid group $\mathbf{B}_{n}$, for some suitable $n$. $\mathbf{B}_{n}$ has $n$ generators, denoted by 


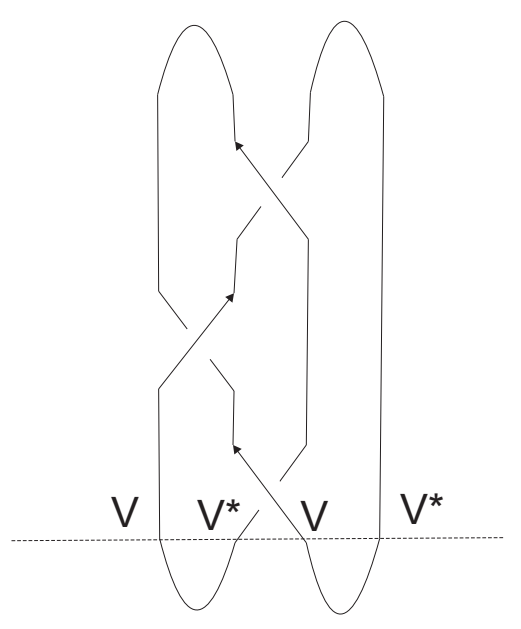

Figure 6: The oriented trefoil knot cut by an horizontal line. We associate with the ordered set of the intersection points (from left to right) the tensor product $V \otimes V^{*} \otimes V \otimes V^{*}$, where each factor is chosen in order to comply with the diagram orientation.

$\left\{\sigma_{1}, \sigma_{2}, \ldots, \sigma_{n-1}\right\}$ plus the identity $e$, which satisfy the relations

$$
\begin{gathered}
\sigma_{i} \sigma_{j}=\sigma_{j} \sigma_{i} \quad \text { if }|i-j|>1 \quad(i, j=1,2, \ldots, n-1) \\
\sigma_{i} \sigma_{i+1} \sigma_{i}=\sigma_{i+1} \sigma_{i} \sigma_{i+1} \quad(i=1,2, \ldots, n-2) .
\end{gathered}
$$

An element of the braid group is a word in the standard generators of $\mathbf{B}_{n}$, e.g. $b=\sigma_{3}^{-1} \sigma_{2} \sigma_{3}^{-1} \sigma_{2} \sigma_{1}^{3} \sigma_{2}^{-1} \sigma_{1} \sigma_{2}^{-2} \in \mathbf{B}_{4}$; the length $|b|$ of the word $b$ is the number of its letters. The group acts naturally on topological sets of $n$ disjoint strands - ordered from left to right - in the sense that each generator $\sigma_{i}$ corresponds to the over-crossing of the $i$ th strand on the $(i+1)-$ th, and $\sigma_{i}^{-1}$ represents the inverse operation (under-crossing) according to $\sigma_{i} \sigma_{i}^{-1}$ $=\sigma_{i}^{-1} \sigma_{i}=e$.

On the other hand, when we represent $\mathbf{B}_{n}$ in the tensor algebra of $U_{q}(\mathfrak{g})$, the action of a braid $b$ is naturally associated with a linear operator $T(b)$ connecting the vector spaces introduced above, see Fig. 7 .

Since $T$ is a linear representation, we can simply specify its action on the standard generators $\left\{\sigma_{1}, \sigma_{2}, \ldots, \sigma_{n-1}\right\}$ to get $\left\{T\left(\sigma_{1}\right), T\left(\sigma_{2}\right), \ldots, T\left(\sigma_{n-1}\right)\right\}$, and extend this action to $T(b)$ by linearity. The $R$-matrix, namely the linear 


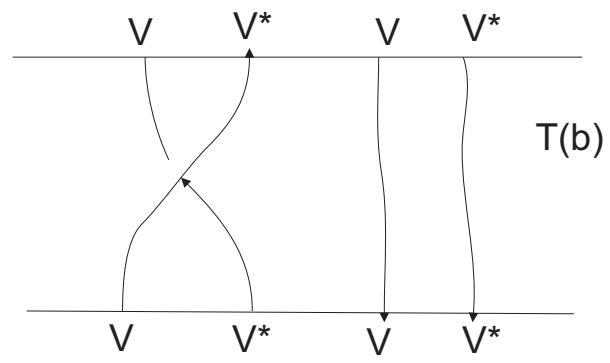

Figure 7: The action of the braid group element $b$ is represented as a map $T(b)$ between the vector spaces living on the bottom and top lines.

operator introduced in (17), is to be intended as the set of (elementary) crossing operators in some given representation $T$, constrained by the quantum Yang-Baxter relation (18).

Knot theory is closely related to (representations of) braid groups owing to Alexander's theorem [28, which states that every knot (or link ) $L$ in the 3 -sphere $S^{3}=\mathbb{R}^{3} \cup \infty$ can be presented (not uniquely) as a closed braid for some suitable $n$ (to get a knot from the open braid of Fig. [7 we have to connect with arcs the lower and upper endpoints of each strand). We might also consider the plat presentation of a knot (characterized by the fact that the braid involved must possess an even number of strands), which is exactly the type of presentation depicted in Fig. 6] for the trefoil knot (see also Fig. 1 and Fig. 11]in the Appendix). Anyway, we can generate invariants of knots (links) for both types of presentations by taking some 'trace' of the operator $T(b)$, where $b$ is the braid associated with the given knot or link. The fact that the resulting quantities must depend only on the isotopy type of the knot can be suitably translated into the braid group-setting by resorting to the notion of invariance under Markov moves, and thus we should actually speak of 'Markov traces' ( $c f r$. [15, 29] for reviews on knot theory and braid group).

Summing up, the quantum group approach provides a purely algebraic construction of link invariants as (Markov) traces of representation matrices of the braid group in the tensor algebra of $U_{q}(\mathfrak{g})$. Such invariants are polynomials in the deformation parameter $q$ and its inverse $1 / q$. In the case of $U_{q}(s u(2))$ ( $q$ a root of unity), the associated $q$-braided Racah-Wigner algebra (discussed in section 4) is naturally endowed with Hilbert spaces and uni- 
tary operators, namely the ideal arena to address (quantum) computational problems concerning both link polynomials and braid group.

\subsection{The Chern-Simons field theory approach}

A topological quantum field theory (TQFT) is a particular type of gauge theory, namely a theory quantized through the (Euclidean) path integral prescription starting from a classical Yang-Mills action defined on a suitable $D$-dimensional space(time). TQFT are characterized by observables (correlation functions) which depend only on the global features of the space on which these theories live, namely they are independent of any metric which may be used to define the underlying classical theory. The geometrical generating functionals and correlation functions of such theories are computable by standard techniques in quantum field theory and provide novel representations of certain global invariants (for $D$-manifolds and/or for particular submanifols embedded in the ambient space) which are of prime interest. Let us recall in brief the basic axioms for a unitary TQFT in $D=3$ before going through the case which is of interest here, namely $S U(2)$ Chern-Simons theory 9 .

Denote by $\Sigma_{1}$ and $\Sigma_{2}$ a pair of 2 -dimensional manifolds and by $\mathcal{M}^{3}$ a 3-dimensional manifold with boundary $\partial \mathcal{M}^{3}=\Sigma_{1} \cup \Sigma_{2}$ (all manifolds here are compact, smooth and oriented). A unitary 3-dimensional quantum field theory corresponds to the assignment of

i) finite dimensional Hilbert spaces (endowed with non-degenerate bilinear forms) $\mathcal{H}_{\Sigma_{1}}$ and $\mathcal{H}_{\Sigma_{2}}$ to $\Sigma_{1}$ and $\Sigma_{2}$, respectively;

ii) a map (technically, a functor) connecting such Hilbert spaces

$$
\mathcal{H}_{\Sigma_{1}} \stackrel{\mathbf{Z}\left[\mathcal{M}^{3}\right]}{\longrightarrow} \mathcal{H}_{\Sigma_{2}}
$$

where $\mathcal{M}^{3}$ is a manifold which interpolates between $\Sigma_{1}$ (incoming boundary) and $\Sigma_{2}$ (outgoing boundary). Without entering into details concerning a few more axioms (diffeomorphism invariance, factorization etc.) we just recall that unitarity implies that

iii) if $\bar{\Sigma}$ denotes the surface $\Sigma$ with the opposite orientation, then $\mathcal{H}_{\bar{\Sigma}}=\mathcal{H}_{\Sigma}^{*}$, where $*$ stands for complex conjugation;

iv) the mappings (20) are unitary and $\mathbf{Z}\left[\overline{\mathcal{M}}^{3}\right]=\mathbf{Z}^{*}\left[\mathcal{M}^{3}\right]$, where $\overline{\mathcal{M}}^{3}$ denote the manifold with the opposite orientation with respect to $\mathcal{M}^{3}$. 
The classical $S U(2)$ Chern-Simons action for the sphere $S^{3}$ (which is the simplest compact, oriented 3-manifold without boundary) is given by

$$
k S(A)=\frac{k}{4 \pi} \int_{S^{3}} \operatorname{tr}\left(A d A+\frac{2}{3} A \wedge A \wedge A\right)
$$

where $A$ is the connection 1 -form with value in the Lie algebra $s u(2)$ of the gauge group, $k$ is the coupling constant, $d$ is the exterior differential, $\wedge$ is the wedge product of differential forms and the trace is taken over Lie algebra indices. The partition function of the quantum theory corresponds to the map (20) restricted to the case of empty boundaries and is obtained as a 'path integral', namely by integrating the exponential of $i$ times the classical action (21) over the space of gauge-invariant flat $S U(2)$ connections (the field variables) according to the formal expression

$$
\mathbf{Z}_{C S}\left[S^{3} ; k\right]=\int[D A] \exp \left\{\frac{i k}{4 \pi} S_{C S}(A)\right\}
$$

where the coupling constant $k$ is constrained to be a positive integer by the gauge-invariant quantization procedure and is related to the deformation parameter $q$ (see below). The generating functional (22), written for a generic compact oriented 3 -manifold $\mathcal{M}^{3}$ with $\partial \mathcal{M}^{3}=\emptyset$, is a global invariant, namely depends only on the topological type [10].

The extension of (22) to the case of a manifold with boundaries, $\partial \mathcal{M}^{3} \neq \emptyset$, requires modifications of the classical action (21) by suitable Wess-Zuminotype terms to be associated with each boundary component 30. However, we do not need here the explicit expression of such boundary action since what we are interested in are expectation values of observables in the quantized field theory which will just require the knowledge of (vectors belonging to) the boundary Hilbert spaces, cfr. i) above. In particular, it turns out that the gauge-invariant observables in the quantum CS theory are expectation values of Wilson line operators associated with oriented knots (links) embedded in the 3-manifold (commonly referred to as Wilson loop operators). Knots and link are 'colored' with irreps of the gauge group $S U(2)$, restricted to values ranging over $\{0,1 / 2,1,3 / 2, \ldots, k-2\}$, where the integer $k$ is related to the deformation parameter $q$ by $q=\exp (-2 i \pi / k)$ (see section 4 for details on the $U_{q}(s u(2))$ representation algebra).

The Wilson loop operator associated with a knot $K$ carrying a spin $-j$ irreducible representation is defined as (the trace of) the holonomy of the 
connection 1-form $A$ evaluated along the closed loop $K \subset S^{3}$, namely

$$
\mathbf{W}_{j}[K]=t r_{j} P \exp \oint_{K} A
$$

where $P$ is the path ordering. For a link $L$ made of a collection of $s$ knots $\left\{K_{l}\right\}$, each labelled by an irrep, the expression of the composite Wilson operator reads

$$
\mathbf{W}_{j_{1} j_{2} \ldots j_{s}}[L]=\prod_{l=1}^{s} \mathbf{W}_{j_{l}}\left[K_{l}\right]
$$

In the framework of the path integral quantization procedure, expectation values of observables are defined as functional averaging weighed with the exponential of the classical action. In particular, the functional average of the Wilson operator (24) is

$$
\mathcal{E}_{j_{1} \ldots j_{s}}[L]=\frac{\int[D A] \mathbf{W}_{j_{1} \ldots j_{s}}[L] e^{\frac{i k}{4 \pi} S_{C S}(A)}}{\int[D A] e^{\frac{i k}{4 \pi} S_{C S}(A)}},
$$

where $S_{C S}(A)$ is the CS action for the 3-sphere given in (21) and the generating functional in the denominator will be normalized to 1 in what follows. It can be shown that this expectation value, which essentially ${ }^{1}$ coincides with the extended (colored) Jones polynomial [11, 12, depends only on the isotopy type of the oriented link $L$ and on the set of irreps $\left\{j_{1}, \ldots, j_{s}\right\}$ (note also that $\mathcal{E}[L]=\mathcal{E}[\bar{L}]$, where $\bar{L}$ is obtained from $L$ by reversing the orientation).

The explicit evaluation of (25) can be carried out in several ways, by resorting to either field-theoretic methods, quantum group approaches (outlined above) or through combinatorial state sum functionals. For future convenience we just sketch here the approach which relies on the extension of CS quantum theory -endowed with a Wess-Zumino conformal field theory

\footnotetext{
${ }^{1}$ These polynomials are actually invariants of 'regular' isotopy, which represents a restricted form of 'ambient' isotopy defined at the beginning of this section. The connection between $\mathcal{E}_{j_{1} \ldots j_{s}}[L]$ and the genuine colored Jones polynomial is given by $J_{j_{1} \ldots j_{s}}\left(L, q^{1 / 2}\right)=$ $\left\{q^{-3 w(L) / 4} /\left(q^{1 / 2}-q^{-1 / 2}\right)\right\} \mathcal{E}_{j_{1} \ldots j_{s}}[L]$, once suitable normalizations for the unknots have been chosen. Here $w(L)$ is the writhe associated with the planar diagram $D(L)$ of the link $L$, defined as $w(L)=\sum_{p} \varepsilon(p)$. The summation runs over the self crossing points of $D(L)$ and $\varepsilon(p)= \pm 1$ according to simple combinatorial rules (see e.g. [27]). The writhe is easily recognized from the link diagram by simple counting arguments, so that computational problems involving both link invariants belong to the same complexity class.
} 
on its boundary - to the case in which the boundary components are intersected by knots or links, namely become 2-manifolds with punctures (note that this setting is closely related to the topological quantum computation approach [5]). The basic geometric ingredients can be easily visualized as in Fig. 8, where a portion of a 3 -dimensional manifold $\mathcal{M}^{3}$ (technically, a handlebody decomposition) is shown, together with an incoming boundary $\Sigma_{1}$ and an outgoing boundary $\Sigma_{2}$ made of two disjoint components, $\Sigma_{2}^{\prime}$ and $\Sigma_{2}^{\prime \prime}$. A portion of some knot (link) embedded in the ambient 3-manifold is also depicted, and its intersections with the boundaries are 'punctures' which inherit the irreps labels from the associated (Wilson) lines.

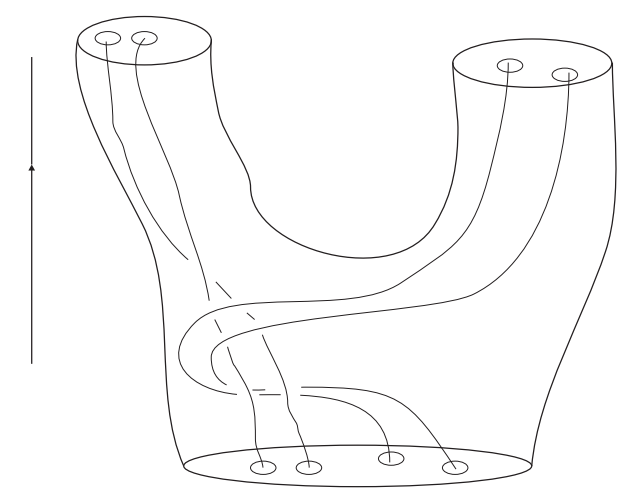

Figure 8: A portion of an oriented 3-manifold with one incoming boundary and two outgoing boundaries. Lines belong to some knot (or link) embedded in the manifold and intersect the $2 D$ boundaries in some points (punctures).

According to the axioms of TQFT, we may associate with each boundary a (finite-dimensional) Hilbert space, that is $\mathcal{H}_{\Sigma_{1}}$ for the incoming boundary and $\mathcal{H}_{\Sigma_{2}} \doteq \mathcal{H}_{\Sigma_{2}^{\prime}} \otimes \mathcal{H}_{\Sigma_{2}^{\prime \prime}}$ (here, for simplicity, we do not explicitate the labels of puncures). The Chern-Simons unitary functional (see axiom ii)) is a state in the tensor product of these Hilbert spaces or, more precisely,

$$
\begin{gathered}
\mathbf{Z}_{C S}\left[\mathcal{M}^{3} ; k\right]: \mathcal{H}_{\Sigma_{1}} \rightarrow \mathcal{H}_{\Sigma_{2}} \\
\Rightarrow \mathbf{Z}_{C S}\left[\mathcal{M}^{3} ; k\right] \in \mathcal{H}_{\Sigma_{1}} \otimes \mathcal{H}_{\Sigma_{2}}^{*},
\end{gathered}
$$

where in the last row we have used also axiom iii) since the incoming and outgoing boundaries must be endowed with opposite orientations. Moreover, 
such type of expression is compatible with the quantum group approach outlined in section 5.1 since the the Chern-Simons mapping in (26), when restricted to punctures, induces automatically (unitary) representations of the braid group in the tensor algebra of $U_{q}(s u(2))$.

Finally, it can be shown [9] that the conformal blocks of the $S U(2)_{\ell}$ WessZumino field theory living on the boundaries with punctures actually provide the basis vectors for the Hilbert spaces introduced above (the level $\ell$ of the WZ model is related to the deformation parameter $q$ according to $q=\exp \{-2 \pi i /(\ell+2)\}$, and in turn $\ell$ is related to the coupling constant $k(\geq 3)$ of the CS theory in the bulk by $\ell=k-2)$. In the following section we shall carry on the explicit construction of such bases, which will allow us to recast the expectation value of the composite Wilson operator (25) into a form suitable to be handled for computational purposes.

\section{Automaton calculation of extended Jones polynomials}

As anticipated at the end of the previous section, we start with the construction of the basis vectors which will enter into the explicit expression of the expectation value of the composite Wilson operator (25) emerging from quantized $3 D$ Chern-Simons theory. We use here the general setting given in 31] since it can be easily adapted to the $q$-braided spin-network scheme of section 4.

Consider an oriented link $L$ embedded in the 3 -sphere, $S^{3}=\mathbb{R}^{3} \cup \infty$, endowed with a plat representation, namely presented as the closure of an oriented braid with $2 N$ strands ( $c f r$. Figg. 11 6] and 11). If we remove two open three-balls from $S^{3}$ we get two boundaries, $\Sigma_{1}$ and $\Sigma_{2}$, both topologically equivalent to $S^{2}$, but with opposite orientations, $\left(S^{3} ; \Sigma_{1}, \Sigma_{2}\right) \equiv$ $\left(S^{3} ; S^{2}, \bar{S}^{2}\right)$ (recall from section 5.2 that an $S U(2)_{\ell}$ Wess-Zumino conformal field theory is naturally associated with the oriented boundary surfaces). We can accomodate in such an ambient manifold, $2 N$ 'unbraided' Wilson lines carrying irreps $j_{1}, j_{2}, \ldots, j_{2 N}$, starting from the incoming (lower) boundary and ending into the outgoing (upper) one (punctures inherit the labellings $j_{i} \in\{0,1 / 2,1,3 / 2, \ldots \ell\}$ from the strands of the braid). Denote this 'identity' 
colored oriented braid as

$$
\nu_{I}\left(\begin{array}{cccc}
\widehat{j}_{1}^{*} & \hat{j}_{2}^{*} & \ldots & \widehat{j}_{2 N}^{*} \\
\hat{j}_{1} & \hat{j}_{2} & \ldots & \widehat{j}_{2 N}
\end{array}\right),
$$

where $\hat{j}_{i} \equiv\left(j_{i}, \epsilon_{i}\right) i=1,2, \ldots 2 N$ represents the spin $j_{i}$ together with an orientation $\epsilon_{i}= \pm 1$ for a strand going into or away from the boundary, while stars over the symbols represent here the opposite choice of the orientation. In order to generate an arbitrary (oriented) braid $\nu_{B}$ out of the identity braid $\nu_{I}$ we have to apply a braiding operator, denoted by the symbol $B$ and written in terms of generators $B_{1}, B_{2}, \ldots, B_{2 N-1}$ to be defined below, starting from the lower boundary. With such prescription we shall get the braid

$$
\nu_{B}\left(\begin{array}{ccccc}
\widehat{j}_{1} & \hat{j}_{1}^{*} & \ldots & \widehat{j}_{N} & \widehat{j}_{N}^{*} \\
\widehat{l}_{1} & \widehat{l}_{1}^{*} & \ldots & \widehat{l}_{N} & \widehat{l}_{N}^{*}
\end{array}\right),
$$

where the labels have been ordered according to the requirement of having a plat presentation for the associated oriented link.

Our goal will consist in recasting the expectation value of the composite Wilson operator, written in functional terms in (25), into an expression which contains a quantity of the type

$$
\left\langle\phi\left|B\left(\begin{array}{ccccc}
\widehat{j}_{1} & \widehat{j}_{1}^{*} & \ldots & \widehat{j}_{N} & \widehat{j}_{N}^{*} \\
\widehat{l}_{1} & \widehat{l}_{1}^{*} & \ldots & \widehat{l}_{N} & \widehat{l}_{N}^{*}
\end{array}\right)\right| \tilde{\phi}\right\rangle,
$$

where $B(:::)$ is the operator associated with the oriented braid (28). The shorthand notations $\mid \phi>$ and $\mid \tilde{\phi}>$ represent correlators of $2 N$ primary fields in the $S U(2)_{\ell}$ WZ theory, to be interpreted here as states belonging to the boundary Hilbert spaces associated, respectively, with the incoming and outgoing Hilbert spaces of the underlying CS theory ( $c f r$. the axioms for TQFT in section 5.2).

The basis vectors to be associated with the incoming boundary can be denoted in general as

$$
\left|\left[j_{1}, j_{2}, \ldots, j_{2 N-1}, j_{2 N}\right] ;[\mathbf{k} ; \mathbf{h}] ; 0,0\right\rangle,
$$

where the last two entries are the quantum numbers $J M \equiv(00)$ for a singlet state of the total angular momentum and the first string represents the incoming spin variables (we drop the hat on oriented objects whenever 
not necessary). The second group of entries denotes particular sets of intermediate angular momentum labels, arising from binary couplings of the $2 N$ primary fields $j$ 's, with a bipartite structure represented by the symbols $\mathbf{k}=k_{1}, k_{2}, \ldots$ and $\mathbf{h}=h_{1}, h_{2}, \ldots$, chosen in order to comply with the rules described below. Before addressing the latter in general, let us have a look at the simplest non trivial case of $N=4$ incoming spin labels. The underlying admissible combinatorial structures are depicted in Fig. 9]

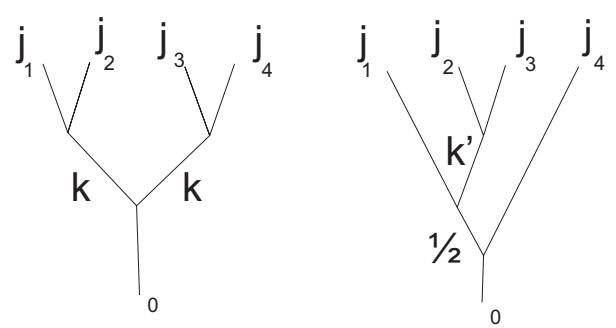

Figure 9: Combinatorial realization of the two basis sets in the case $N=4$ as labelled binary trees. They are connected by a duality matrix.

In this case we have just two types of basis vectors, related by the socalled duality matrix of WZ theory

$$
\left|\left[j_{1}, j_{2}, j_{3}, j_{4}\right] ;[k, k ;-] ; 00\right\rangle=\sum_{k^{\prime}}\left(\begin{array}{cc|c}
j_{1} & j_{2} & k \\
j_{3} & j_{4} & k^{\prime}
\end{array}\right)_{q}\left|\left[j_{1}, j_{2}, j_{3}, j_{4}\right] ;\left[k^{\prime} ; \frac{1}{2}\right] ; 00\right\rangle,
$$

where the vectors on the left hand side do not contain any $\mathbf{h}$-label, namely $[\mathbf{k}, \mathbf{h}]=[k, k ;-]$, while on the right we have the combination $\left[\mathbf{k}^{\prime}, \mathbf{h}^{\prime}\right]=\left[k^{\prime} ; \frac{1}{2}\right]$. The array $(:: \mid:)_{q}$ in (31) is the (normalized) $q$-Racah symbol of $U_{q}(s u(2))$ introduced in (16) of section 4 (with respect the notation used there, from now on we drop the subscript $q$ on vectors).

In the general case of $2 N$ incoming spin labels, the two combinatorially distinct bases which have to be involved are specializations of the vectors in (30) to the two configurations depicted in Fig. 10. The extension of the duality transformation (31) to the case of an arbitrary (even) number of incoming spins can be done by resorting to two types of more complicated 

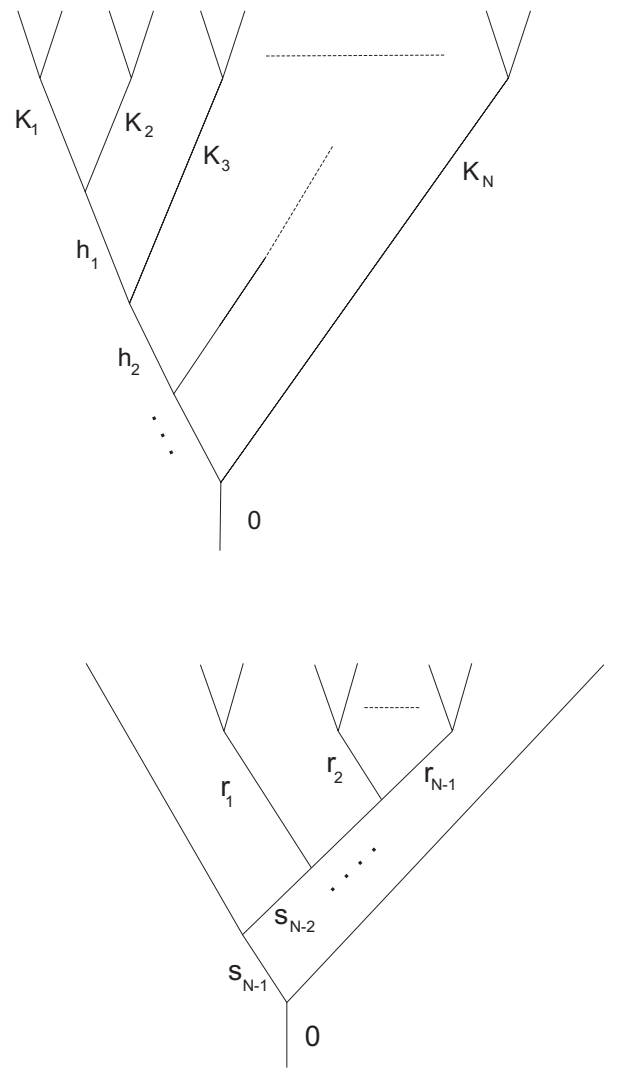

Figure 10: Coupling binary trees representing the combinatorics of the two sets of bases in the case of a generic $N$, see (35) and (34).

arrays, which can be represented as

$$
\left(\begin{array}{cc|cc}
j_{1} & j_{2} & k_{1} & h_{1} \\
j_{3} & j_{4} & k_{2} & h_{2} \\
\vdots & \vdots & \vdots & \vdots \\
j_{2 N-5} & j_{2 N-4} & k_{N-2} & h_{N-2} \\
j_{2 N-3} & j_{2 N-2} & k_{N-1} & - \\
j_{2 N-1} & j_{2 N} & k_{N} & -
\end{array}\right)_{q},\left(\begin{array}{ccc|cc}
j_{2} & j_{3} & r_{1} & s_{1} \\
j_{4} & j_{5} & r_{2} & s_{2} \\
\vdots & \vdots & \vdots & \vdots \\
j_{2 N-4} & j_{2 N-3} & r_{N-2} & s_{N-2} \\
j_{2 N-2} & j_{2 N-1} & r_{N-1} & s_{N-1} \\
j_{2 N} & j_{1} & - & -
\end{array}\right)_{q}
$$

where the matrix indices - to be involved in summations whenever transformations which generalize (31) are implemented- are listed in the right hand 
side of the arrays.

As happens in the standard Racah-Wigner setting, it can be shown that each of these arrays, which represent the $q$-deformed counterparts of $S U(2) 3 n j$ coefficients, can be decomposed in terms of $q$-Racah transformations (31), cfr. sections 3,4 of [2] and [31].

When the outgoing Hilbert space is considered (corresponding to the boundary $\Sigma_{2}$ endowed with the opposite orientation with respect to $\Sigma_{1}$ ) we have to introduce bra-type bases which are dual (and orthonormal) with respect to the bases in (30). With an obvious choice of notations we set

$$
\left\langle\left[j_{1}, \ldots, j_{2 N}\right] ;[\mathbf{k} ; \mathbf{h}] ; 0,0 \mid\left[j_{1}, \ldots, j_{2 N}\right] ;\left[\mathbf{k}^{\prime} ; \mathbf{h}^{\prime}\right] ; 0,0\right\rangle=\delta_{\mathbf{k}, \mathbf{k}^{\prime}} \delta_{\mathbf{h}, \mathbf{h}^{\prime}},
$$

where, as before, $\mathbf{h}, \mathbf{k}, \mathbf{h}^{\prime}, \mathbf{k}^{\prime}$ represent multi-indices to be associated with the admissible configurations of binary coupled spins and there appear multiple Kronecker deltas.

The discussion above was aimed to recognizing the crucial fact that the basis vectors

$$
\left|\left[j_{1}, j_{2}, \ldots, j_{2 N-1}, j_{2 N}\right] ;\left[k_{1}, \ldots, k_{N} ; h_{1}, \ldots, h_{N-2}\right] ; 0,0\right\rangle
$$

are eigenfunctions of the odd braiding operators $B_{2 l-1}$, while the basis vectors

$$
\left|\left[j_{1}, j_{2}, \ldots, j_{2 N-1}, j_{2 N}\right] ;\left[r_{1}, \ldots, r_{N-1} ; s_{1}, \ldots, s_{N-1}\right] ; 0,0\right\rangle
$$

are eigenfunctions of the even braiding operators $B_{2 l}$.

The explicit expressions of the eigenvalues, in the odd and even case respectively, read

$$
\begin{gathered}
\lambda_{k_{l}}\left(\hat{j}_{2 l-1}, \hat{j}_{2 l}\right) \doteq \lambda_{z}^{(+)}\left(j, j^{\prime}\right)=(-)^{j+j^{\prime}-z} q^{\left(c_{j}+c_{j^{\prime}}\right) / 2+c_{\min \left(j, j^{\prime}\right)}-c_{z} / 2} \text { for } \epsilon \epsilon^{\prime}=+1 \\
\lambda_{r_{l}}\left(\hat{j}_{2 l}, \hat{j}_{2 l+1}\right) \doteq\left(\lambda_{z}^{(-)}\left(j, j^{\prime}\right)\right)^{-1}=(-)^{\left|j-j^{\prime}\right|-z} q^{\mid\left(c_{j}-c_{j^{\prime}} \mid / 2-c_{z} / 2\right.} \text { for } \epsilon \epsilon^{\prime}=-1 .
\end{gathered}
$$

Here $l=1,2, \ldots, N-1, q$ is the deformation parameter, $z \in\left\{k_{1}, k_{2}, \ldots, k_{N}\right.$, $\left.r_{1}, r_{2}, \ldots, r_{N-1}\right\}, c_{z} \equiv z(z+1)$ is the quadratic Casimir for the spin- $z$ representation and $\epsilon, \epsilon^{\prime}$ denote the orientation of the strands labelled by $j$ and $j^{\prime}$, respectively. Thus $\lambda_{z}^{(+)}\left(j, j^{\prime}\right)$ is the eigenvalue of the matrix which performs a right handed half-twist in contiguous strands with the same orientation, while $\lambda_{z}^{(-)}\left(j, j^{\prime}\right)$ is the eigenvalue of the matrix which performs a right handed half-twist in strands with opposite orientation. 
The explicit expression of the formal expectation value given in (29) above gives, after normalization according to the standard conventions ( $c f r$. 31]), the extended Jones polynomial of the colored link $L$ associated with the braid (28), namely

$$
\begin{aligned}
& V_{j_{1} j_{2} \ldots j_{N}}[L ; q]=\prod_{i=1}^{N}\left[2 j_{i}+1\right]_{q} \times \\
& \left\langle\left[\hat{l}_{1}, \hat{l}_{1}^{*}, \ldots, \hat{l}_{N}, \hat{l}_{N}^{*}\right] ;[0 ; 0] ; 0,0\left|B\left(\begin{array}{ccccc}
\hat{j}_{1} & \hat{j}_{1}^{*} & \ldots & \hat{j}_{N} & \hat{j}_{N}^{*} \\
\hat{l}_{1} & \hat{l}_{1}^{*} & \ldots & \hat{l}_{N} & \hat{l}_{N}^{*}
\end{array}\right)\right|\left[\hat{j}_{1}, \hat{j}_{1}^{*}, \ldots, \hat{j}_{N}, \hat{j}_{N}^{*}\right] ;[0,0] ; 0,0\right\rangle,
\end{aligned}
$$

where $\left[2 j_{i}+1\right]_{q}$ is the $q$-dimension of the $U_{q}(s u(2))$ irrep $j_{i}$ defined in section 4. The operator $B(:::)$ is expressed in terms of (a finite sequence of) the elementary braiding operators $\left\{B_{2 l-1} ; B_{2 l}\right\}$, suitably changed into the current odd (even) basis by acting with a $q$-duality matrix, whenever an even (odd) vector of type (35) (134), respectively) is encountered. According to the expressions (32) and (36) for the admissible operations, the running variable of the polynomial is given by $q=\exp \{-2 \pi i /(\ell+2)\}$ for any integer $\ell \geq 3$. Moreover, the above expectation value is to be interpreted as a trace over free spin labels. This feature derives of course from the geometric construction of the plat presentation of the link $L$ outlined at the beginning of this section, since the colored oriented braid (28) has to be 'closed up' to get the associated link. More precisely, corresponding strands must not only match pairwise with the correct orientations (as is made manifest by our notation), but $j$ and $l$-type labels have to be appropriately identified (traced) in pairs, namely the l's in (37) are not new independent labels.

Coming back to the 3-dimensional picture, such trace procedure amounts to gluing back the two opposite-oriented boundary 2-spheres, paying attention to the coloring of the punctures, to end up with the same 3-sphere we started from. As already pointed out, the resulting link polynomial, arising as vacuum expectation value of the composite Wilson loop operator (25) in the quantum $S U(2) \mathrm{CS}$ theory for $\mathcal{M}^{3}=S^{3}$, is automatically an invariant of regular isotopy ( $c f r$. the remarks in the footnote of section 5.2).

This long technical discussion about the derivation of the extended Jones polynomial is nothing but the necessary premise to address the main issue of the present paper, namely the analysis of the connections among the theory of formal languages (section 2) and the spin-network computational scheme (sections 3 and 4), on the one hand, and braid group and links invariants on 
the other. We are going to interpret the results established so far in terms of 'processing of words', written in the alphabet given by the generators of the braid group, on a quantum automaton in such a way that the expectation value associated with the 'evolution' of the automaton is precisely the extended Jones polynomial. The quantum automaton in question will in turn correspond to a path in the $q$-braided spin-network computational graph.

In order to comply with the requirements for a finite-states quantum automaton described in section 2, we have to provide explicitly the 5-tuple $\left(Q, \Sigma, \delta, \mathbf{q}_{0}, F\right)$, where $F$ represents a set of acceptable final states. Now $Q$ is a finite set of states belonging to the Hilbert spaces of the tensor algebra of $U_{q}(s u(2))$ described in section 5.1, whose combinatorial content was depicted in Fig. 10. Labels of $N$ irreps in this quantum group are associated with the strands of the plat presentation of the link $L$, and $\mathbf{B}_{2 N}$ is the braid group to be selected. $\Sigma$ is the alphabet made of the $2 N-1$ generators of $\mathbf{B}_{2 N}$ : each generator (and its inverse) represents a letter of the alphabet, and words are written as composition of these elementary braids. The function $\delta$ denotes a set of unitary matrices defining the transition rules and there is of course one matrix for each letter of the alphabet since we are linearly representing the braid group in the tensor algebra $U_{q}(s u(2))$.

As initial state $\mathbf{q}_{0}$ we pick up one particular binary-coupled state, namely

$$
\left|\left[\left(j_{1}, j_{2}\right), \ldots,\left(j_{2 N-1}, j_{2 N}\right)\right] ;[0 ; 0] ; 0,0\right\rangle,
$$

where, with respect to the generic expression for an odd basis vector given in (134), we choose $[\mathbf{k} ; \mathbf{h}]=[0 ; 0]$, namely we select a 'multi-singlet' intermediate state.

The set of final states $F$ is constrained by the topological properties of the plat presentation, namely final states may differ from $\mathbf{q}_{0}$ by a permutation on the string $\left(j_{1} j_{2} \ldots j_{2 N}\right)$. Thus we can actually build up a family of $N$ ! automata out of one initial state $\mathbf{q}_{0}$. By acting with the symmetric group on the binary parenthesization structure of (38) we may get, for instance, the singlet final state

$$
\left.\left.\mid\left[\left(\left(\left(\left(j_{3}, j_{2}\right),\left(j_{1}, j_{4}\right)\right),\left(j_{2 N}, j_{6}\right)\right), \ldots\right),\left(j_{2 N-1}, j_{5}\right)\right)\right] ;[0,0] ; 0,0\right\rangle .
$$

The unitary transition rules codified in the set $\delta$ are:

- if the automaton is in an even (odd) state and it reads an even (odd) braid generator, then the system evolves with the $\mathrm{R}$-matrix associated to the proper braid generator, $B_{2 l-1}$ or $B_{2 l}$, see (34), (35) and (36); 
- if the automaton is in an odd (even) state and it reads an even (odd) braid generator, then the system evolves with the proper duality transformation (see (32)) to update the actual state into the configuration consistent with the parity of the given braid generator. As pointed out before, such a transformation can be splitted into a finite sequence of elementary duality ( $q$-Racah) transformations of the type (31).

Once a final state $\mathbf{q}_{f}$ has been selected (the right permutation can be singled out in a fast way even by a classical machine) the evaluation of the polynomial (37) is carried out by the automaton in a number of steps linear in the length $|B|$ of the 'word' $B$. Since $|B|$ is the sum of the numbers of elementary braiding operators and $q$-duality transformations entering the explicit expression of $B$, the length of the word is bounded from above by a linear function of the number of crossings of the plat presentation of the associated link $L$. On the other hand, in the worst case we have to perform one duality transformation (32) before applying each elementary braiding operator $B_{i} \in\left\{B_{2 l-1}, B_{2 l}\right\}$. This happens, for instance, in the evaluation of the Jones polynomial of the trefoil knot illustrated in the Appendix. This latter remark lead us to conclude that the time complexity function (more precisely, the number of computational steps in our automaton calculation) equals the length of $B$ and is bounded according to

$$
|B| \leq \mathfrak{c}(N) \kappa(L),
$$

where $\mathfrak{c}(N)$ is a positive number depending on the number of generators of the braid group $\mathbf{B}_{2 N}$ and $\kappa(L)$ is the number of crossings in the plat presentation of the link $L$.

We can estimate $\mathfrak{c}(N)$ by observing that any such automaton can be uniquely associated with a particular path $\mathcal{P}$ in the $q$-braided spin-network computational space $\left(\mathfrak{G}_{n}(V, E) \times \mathbb{C}^{2 J+1}\right) \times \mathbb{Z}_{2}$ for $n=2 N-1$. In particular, the maximum number of elementary $q$-Racah transforms entering a duality matrix of type (32) must coincide with the number of Racah transforms entering into one (classical) $3 n j$ symbol since the combinatorics of such operations is manifestly the same. Hence we may exploit results from graph theory which tell us that the Rotation graph $\tilde{\mathfrak{G}}_{n}(\tilde{V}, \tilde{E})$-obtained from $\mathfrak{G}_{n}(V, E)$ by ignoring twists (or braidings)- has a diameter of the order $n \ln n$ (the diameter is defined as the maximum over the set of distances between pairs of vertices, where the distance is the minimum number of edges connecting two given vertices) (cfr. [32] and appendix A of [2] for a complete discussion 
of the spin-network combinatorics). Clearly the 'distance' between the current basis and the eigenbasis with the right parity cannot exceed the above maximum distance, and consequently the factor $\mathfrak{c}(N)$ in (40) grows as

$$
\mathfrak{c}(N) \sim(2 N-1) \ln (2 N-1),
$$

namely polynomially in the number of strands of the link.

A deeper connection with the $q$-braided spin-network computational scheme comes out however when we recognize that the expectation value (37) representing the extended Jones polynomial is not only the quantum transition amplitude of a finite states-automaton, as pointed out before, but complies also with the expectation value (10) to be associated with a path $\mathcal{P}$ in the $q$-version of the spin-network computational space. In this new perspective, what we are really doing is to 'encode' the combinatorial structure underlying quantum $S U(2)$ Chern-Simons field theory (and the associated WZ boundary theory) at some fixed level $\ell$ into the abstract $q-$ braided $S U(2)$-spin-network for $q=\exp \{-2 \pi /(\ell+2)\}$. This does not mean, of course, that we have set up a quantum algorithm for the extended Jones polynomial in the strict sense, since the encoding map could not be 'efficiently' represented (nor efficiently approximated) with respect to standard models of computation (Boolean circuits, Turing machines). We provide, however, a quantum system whose evolution can be controlled in such a way that its probability amplitudes give the desired link polynomials.

The crucial issue of constructing a bona fide quantum algorithm is under investigation. It will require in particular: i) (efficient) encoding schemes for binary coupled states; ii) (efficient) algorithms to evaluate (or approximate) the basic operations, namely the Racah transform and its associated $6 j-$ symbol for arbitrary entries on the one hand, and the elementary braiding operators on the other.

As a final remark we notice that the field theoretical approach gives us automatically the rate of growth of the absolute value of extended Jones polynomial with respect to the Chern-Simons coupling constant $k=\ell+2$. The absolute value of the Reshetikhin-Turaev [11] quantum invariants of 3manifolds, $\left|Z_{k}^{G}\left(\mathcal{M}^{3}\right)\right|$ (which are linear combinations of colored polynomials associated with surgery framed links $\subset \mathcal{M}^{3}$ ) are estimated to grow as $\mathcal{O}\left(k^{d}\right)$, where the exponent $d$ is bounded from above by some simple function (depending on the gauge group $G$ ) of the Heegaard genus of the manifold ( $c f r$. [33], Ch. 7). 


\section{References}

[1] A. Marzuoli and M. Rasetti (2002), Spin network quantum simulator, Phys. Lett. A306, pp. 79-87.

[2] A. Marzuoli and M. Rasetti (2005), Computing spin network, Ann. Phys. 318, pp. 345-407.

[3] M.H. Freedman, A. Kitaev and Z. Wang (2002), Simulation of topological field theories by quantum computers, Commun. Math. Phys. 227, pp. 587-603.

[4] M.H. Freedman, A. Kitaev and Z. Wang (2002), A modular functor which is universal for quantum computation, Commun. Math. Phys. 227, pp. 605-622.

[5] M.H. Freedman, A. Kitaev, M. Larsen and Z. Wang (2002), Topological quantum computation, Bull. Amer. Math. Soc. 40, pp. 31-38.

[6] L.C. Biedenharn and J.D. Louck (1981) The Racah-Wigner Algebra in Quantum Theory (Topic 12. Coupling of N Angular Momenta: Recoupling Theory), Encyclopedia of Mathematics and its Applications, Vol 9, G-C. Rota Ed., Addison-Wesley Publ. Co. (Reading MA).

[7] V.F.R. Jones (1985) A polynomial invariant for knots via von Neumann algebras, Bull. Amer. Math. Soc. 12, pp. 103-111.

[8] F.Y. Wu (1992) Knot theory and statistical mechanics, Rev. Mod. Phys. 64, n.4, pp. 1099-1131.

[9] M. F. Atiyah (1989) Topological quantum field theories, Publ. Math. IHES 68, pp. 175-186.

[10] E. Witten (1989) Quantum field theory and the Jones polynomial, Commun. Math. Phys. 121, pp. 351-399.

[11] N. Reshetikhin and V. G. Turaev (1991) Invariants of 3-manifolds via link polynomials and quantum groups, Invent. Math. 103, pp. 547-597.

[12] R. Kirby and P. Melvin (1991) The 3-manifold invariants of Witten and Reshetikhin-Turaev for $\operatorname{sl}(2, C)$ Invent. Math. 105, pp. 473-545. 
[13] F. Jaeger, D.L. Vertigan and D.J.A. Welsh (1990) On the computational complexity of the Jones and Tutte polynomials, Math. Proc. Cambridge Phil. Soc. 108, pp. 35-53.

[14] D. Aharonov, V. Jones and Z. Landau (2005) A polynomial quantum algorithm for approximating the Jones polynomial, quant-ph/0511096.

[15] J.S. Birman and T.E. Brendle (2004) Braids: a survey, math./ GT/ 0409205.

[16] I. Anshel, M. Anshel, and D. Goldfeld (1999) An algebraic method for public key cryptography, Math. Res. Lett. 6, pp. 287-291.

[17] S. Garnerone, A. Marzuoli and M. Rasetti (2005) Spin networks, quantum automata and link invariants, gr-qc/0511161.

[18] P. Freyd, D. Yetter, J. Hoste, W. Lickorish, K. Millett and A. Ocneanu (1985) A new polynomial invariants of knots and links, Bull. Amer. Math. Soc. 12, pp. 183-312.

[19] J. E. Hopcroft and J. D. Ullman (1979) Introduction to Automata Theory, Languages and Computation, Addison-Wesley (Reading MA).

[20] A. Ambainis, J. Watrous (2002) Two-way finite automata with quantum and classical states Theoret. Comput. Sci. 287, pp. 299-311.

[21] C. Moore and J.P. Crutchfield (2000) quantum automata and quantum grammars Theor. Comput. Sci. 37, pp. 275-306.

[22] J. Kempe, D. Bacon, D.A. Lidar and K.B. Whaley (2001) Theory of decoherence-free fault-tolerant universal quantum computation Phys. Rev. A 63, 042307-1/29.

[23] V. Aquilanti and C. Coletti (2001) 3nj-symbols and harmonic superposition coefficients: an icosaedral abacus Chem. Phys. Lett. 344, pp. 601-611.

[24] C.Kassel (1994) Quantum Groups Grad. Texts in Math. 155, SpringerVerlag (New York-Heidelberg-Berlin) 
[25] A.N. Kirillov and N.Y. Reshetikhin (1988) Representations of the algebra $U_{q}\left(s l_{2}\right), q$-orthogonal polynomials and invariants of links, in 'Infinite dimensional Lie algebras and groups', Ed. V.G. Kac, Adv. Ser. in Math. Phys. 7, World Scientific (Singapore), pp. 285-339.

[26] E. Guadagnini (1993) The Link Invariants of the Chern-Simons Field theory, de Gruyter (Berlin).

[27] L.H. Kauffman (1991) Knots and physics, World Scientific (Singapore).

[28] J. Alexander (1923) A lemma on a system of knotted curves, Proc. Nat. Acad. Sci. USA, 9, pp. 93-95.

[29] J.S. Birman (1974) Braids, Links and Mapping Class Groups, Princeton Univ. Press (Princeton NJ).

[30] S. Carlip (1998) Quantum Gravity in 2+1 dimensions, Cambridge University Press (Cambridge).

[31] R.K. Kaul (1994) Chern-Simons theory, coloured oriented braids and link invariants, Commun. Math. Phys. 162, pp. 289-319.

[32] V. Fack, S. Lievens and J. Van der Jeugt (2002) On the diameter of the rotation graph of binary coupling trees, Discr. Math. 245, pp. 1-18.

[33] T. Ohtsuki Ed. (2002) Problems on invariants of knots and 3-manifolds, Geometry and Topology Monographs, Vol. 4.

\section{Appendix}

A simple application of the procedure described in section 6 for the evaluation of quantum link invariants is the explicit computation of the Jones polynomial for the plat presentation of the trefoil knot $K_{\text {tref }}$ depicted in Fig. [1]. The four strands are labelled by a same $j_{1}$, together with its opposite $j_{1}^{*}$, from left to right.

Accordingly, the initial and final states to be associated with the quantum automaton are

$$
\left|\left[\left(\left(\left(j_{1}, j_{1}^{*}\right),\left(j_{1}^{*}, j_{1}\right)\right)\right)\right] ;[0,0 ;-] ; 0,0\right\rangle
$$

and

$$
\left|\left[\left(\left(\left(j_{1}^{*}, j_{1}\right),\left(j_{1}, j_{1}^{*}\right)\right)\right)\right] ;[0,0 ;-] ; 0,0\right\rangle
$$




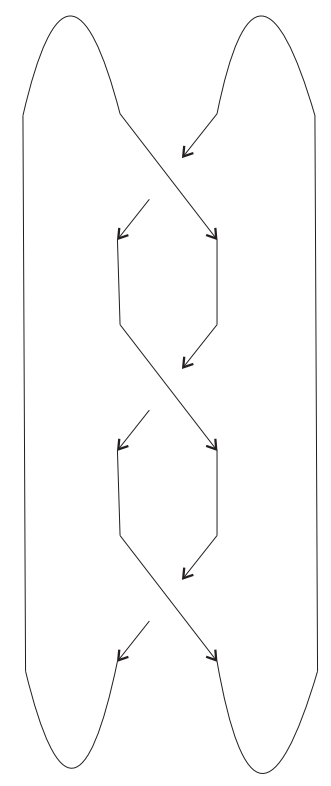

Figure 11: Plat presentation of the oriented trefoil knot.

respectively, and they comply with the prescription of being odd vectors, see (38). From the picture we easily recognize that the operator to be employed is $B_{\text {tref }}=\left(B_{2}\right)^{3}$. Since we are interested in the evaluation of the Jones polynomial we set from now on $j_{1}, j_{1}^{*} \equiv \frac{1}{2}$. Moreover, in order to apply the even braiding operator $B_{2}$ we have to perform preliminarly a duality transformation (31) on the odd vector (42)

$$
\left|\left[\frac{1}{2}, \frac{1}{2}, \frac{1}{2}, \frac{1}{2}\right] ;[0 ;-] ; 00\right\rangle=\sum_{l=0}^{1}\left(\begin{array}{cc|c}
\frac{1}{2} & \frac{1}{2} & l \\
\frac{1}{2} & \frac{1}{2} & 0
\end{array}\right)_{q}\left|\left[\frac{1}{2}, \frac{1}{2}, \frac{1}{2}, \frac{1}{2}\right] ;[l ;-] ; 00\right\rangle,
$$

which converts the initial state of the automaton into eigenvectors of the braid generator $B_{2}$. The application of $B_{\text {tref }}$ gives

$\left(B_{2}\right)^{3}\left|\left[\frac{1}{2}, \frac{1}{2}, \frac{1}{2}, \frac{1}{2}\right] ;[0 ;-] ; 00\right\rangle=\sum_{l=0}^{1}\left(\lambda_{l}^{(+)}\right)^{3}\left(\begin{array}{cc|c}\frac{1}{2} & \frac{1}{2} & l \\ \frac{1}{2} & \frac{1}{2} & 0\end{array}\right)_{q}\left|\left[\frac{1}{2}, \frac{1}{2}, \frac{1}{2}, \frac{1}{2}\right] ;[l ;-] ; 00\right\rangle$,

where there appears the cube of the eigenvalue $\lambda_{l}^{(+)}$defined in (36) . According to the expression of the extended Jones polynomial given in (37) and 
taking into account (43), we get

$$
V_{j=\frac{1}{2}}\left(K_{\mathrm{tref}} ; q\right)=[2]_{q}\left\langle\left[\frac{1}{2}, \frac{1}{2}, \frac{1}{2}, \frac{1}{2}\right] ;[0 ;-] ; 00\left|\left(B_{2}\right)^{3}\right|\left[\frac{1}{2}, \frac{1}{2}, \frac{1}{2}, \frac{1}{2}\right] ;[0 ;-] ; 00\right\rangle,
$$

which, by using the orthogonality relations of the duality matrices (KAUL), amounts to

$$
V_{j=\frac{1}{2}}\left(K_{\mathrm{tref}} ; q\right)=[2]_{q} \sum_{l=0}^{1} \lambda_{l}^{(+)^{3}}=\frac{-1+q+q^{3}}{q^{4}}
$$

as required. 